\title{
Produção de frangos de corte e tecnologias para nutrição, imunologia e melhoramento genético: uma revisão narrativa
}

\author{
Beef poultry production and technologies for nutrition, immunology and genetic \\ improvement: a narrative review
}

Producción y tecnologías de pollos de corral para la nutrición, inmunología y mejoramiento genético: una revisión narrativa

Cátia Borges Ferreira1', Rodrigo Alberto Peixoto Rodrigues de Souza ${ }^{1 \star}$.

\begin{abstract}
RESUMO
Objetivo: Descrever o desenvolvimento da indústria de aves de corte no que diz respeito ao incremento tecnológico implementado para nutrição, imunologia e melhoramento genético. Revisão bibliográfica: $O$ incremento tecnológico utilizado para produção de aves de corte reforça a importância da imunomodulação, utilizando-se a nutrição como resposta rápida e eficaz para melhoria do sistema imunológico das aves, sobretudo, em sua fase inicial de vida. Nesse sentido, há interferência direta na produção, pois, quanto melhor o sistema imunológico, melhor será o desenvolvimento desses animais. Estudos sobre dietas com adequação de vitaminas, minerais, aminoácidos e ácidos graxos são recomendados para melhoria da produtividade, em consonância ao bem-estar e à capacidade imunitária das aves, estimulando a resistência a doenças e eliminando outros problemas, visto que as alterações do sistema imunológico demandam energia e diversos nutrientes para a formação do sistema de defesa aviário. Considerações finais: A imunonutrição é uma forma de otimizar o sistema imunológico dos animais, através do fornecimento dos nutrientes necessários e em quantidades adequadas, para o bom desempenho produtivo e reprodutivo das aves.
\end{abstract}

Palavras-chave: Nutrição, Imunologia, Melhoramento genético, Produção, Frangos de corte.

\begin{abstract}
Objective: To describe the development of the broiler industry with regard to the technological improvement implemented for nutrition, immunology and genetic improvement. Bibliographic review: The technological increment used for the production of broilers reinforces the importance of immunomodulation, using nutrition as a quick and effective response to improve the immune system of birds, especially in their early stages of life. In this sense, there is direct interference in production, as the better the immune system, the better the development of these animals. Studies on diets with adequate vitamins, minerals, amino acids and fatty acids are recommended to improve productivity, in line with the well-being and immune capacity of birds, stimulating resistance to disease and eliminating other problems, as changes in the immune system demand energy and various nutrients for the formation of the avian defense system. Final considerations: Immunonutrition is a way to optimize the animals' immune system, by providing the necessary nutrients and in adequate amounts, for the good productive and reproductive performance of the birds.
\end{abstract}

Key words: Nutrition, Immunology, Genetic improvement, Production, Beef poultry.

\section{RESUMÉN}

Objetivo: Describir el desarrollo de la industria de pollos de engorde con respecto al mejoramiento tecnológico implementado para la nutrición, inmunología y mejoramiento genético. Revisión de la literatura: El incremento tecnológico utilizado para la producción de pollos de engorde refuerza la importancia de la inmunomodulación, utilizando la nutrición como una respuesta rápida y eficaz para mejorar el sistema inmunológico de las aves, especialmente en sus primeras etapas de vida. En este sentido, existe una interferencia directa en la producción, ya que cuanto mejor es el sistema inmunológico, mejor es el desarrollo

\footnotetext{
1 Faculdade Arnaldo Janssen, Belo Horizonte - MG.

*E-mail: rodrigo.peixoto@gmail.com
}

SUBMETIDO EM: 11/2021 
de estos animales. Se recomiendan estudios sobre dietas con adecuadas vitaminas, minerales, aminoácidos y ácidos grasos para mejorar la productividad, en consonancia con el bienestar y la capacidad inmunológica de las aves, estimulando la resistencia a enfermedades y eliminando otros problemas, ya que los cambios en el sistema inmunológico demandan energía. y varios nutrientes para la formación del sistema de defensa aviar. Consideraciones finales: La inmunonutrición es una forma de optimizar el sistema inmunológico de los animales, aportando los nutrientes necesarios y en cantidades adecuadas, para el buen desempeño productivo y reproductivo de las aves.

Palabras clave: Nutrición, Inmunología, Mejoramiento genético, Producción, Avicultura de carne.

\section{INTRODUÇÃO}

Atualmente, a cadeia produtiva de aves de corte evidencia-se no agronegócio brasileiro. Esse aumento da produção advém do uso intensivo de tecnologias que envolvem nutrição, imunologia, manejo das aves e melhoramento genético (BASSI NSS e SILVA CL, 2017). Vasconcelos MC, et al. (2021) relacionam o avanço da produção da carne de frango a crescimento populacional, urbanização, mudanças tecnológicas e organizacionais e hábitos alimentares do consumidor.

Segundo Bassi NSS e Silva CL (2017), o Brasil é um dos principais fornecedores mundiais de carne de aves, e a alta competitividade e o mercado consumidor (nacional e internacional) exigem um esforço contínuo em Pesquisa, Desenvolvimento e Inovação (PD\&l) para manutenção da cadeia produtiva. Portanto, falhas geram impactos desastrosos no desempenho das aves e aumento significativo de custos de produção.

A indústria aviária visa o máximo desempenho das aves, com menor conversão alimentar, saúde intestinal em boas condições, no menor tempo, máximo desempenho da carcaça, organismo das aves em perfeitas condições para a sanidade do lote, sistema imune apto para melhoria contínua na qualidade das aves, minimizando custos e maximizando lucros (MARTINS JMS, et al., 2012). Assim, a ciência e a tecnologia são essenciais para a inovação nos âmbitos de nutrição, manejo, sanidade e genética, além de possibilitar produção sustentável (DEMATTÊ FILHO LC e MARQUES PEM, 2011).

Quanto à genética, Costa LS (2015) ressalva que a produção brasileira ainda depende de pesquisa científica e tecnológica internacional para desenvolvimento e competitividade. Contudo, há grandes perspectivas de aprimoramento contínuo e independente, quanto à nutrição, propiciando ganhos de imunidade para aves de corte.

Neste artigo, o objetivo foi descrever o desenvolvimento da indústria de aves de corte quanto ao incremento tecnológico implementado para nutrição, imunologia e melhoramento genético.

\section{REVISÃO BIBLIOGRÁFICA}

\section{Inovação e Tecnologia no Setor Avícola}

A crescente competitividade do mercado exige que as indústrias (e.g. produção de aves de corte) mostrem vantagens na economia mundial, por meio da inovação (VASCONCELOS MC, et al., 2021). Nesse cenário, destaca-se a produção tecnológica, decorrente do desenvolvimento científico, ofertando conhecimentos fundamentados em pesquisas científicas. Segundo Vasconcelos MC, et al. (2021), a tecnologia abrange conhecimentos científicos e empíricos, habilidades e experiências necessárias para utilizar, produzir e comercializar bens e serviços, contemplando informações teóricas e práticas relacionadas à produção e à gestão.

Assim, é preciso criar novas formas de organização do trabalho e introduzir novos produtos em mercados variados, oferecendo melhoramentos significativos em especificações técnicas, componentes e materiais, facilidade de uso, etc. (VASCONCELOS MC, et al., 2021).

Avanços tecnológicos proporcionam aumento acentuado dos índices agropecuários e as propriedades rurais geram excedentes de consumo para atender aos novos e exigentes mercados. No setor de aves de 
corte, esses avanços envolvem fabricantes de material genético, produtos veterinários e aditivos químicos, inovacão de máquinas e equipamentos responsáveis pelo desenvolvimento dessa indústria (BASSI NSS e SILVA CL, 2017). Posto que, na nutrição animal, a alimentação facilita o ganho de peso, conforme cada linhagem, busca-se manter ganho semanal constante e uniforme das aves (PESSOA GBS, et al., 2012).

Quanto ao mercado de material genético na avicultura, atualmente, as poucas empresas, localizam-se nos Estados Unidos, na França, Holanda, Escócia, entre outros países que fomentam linhagens e repassam o material ao mercado internacional (COSTA LS, 2015).

Nesse mercado, há estratégias de diferenciação de importantes produtos na competição entre indústrias avícolas. Para melhor desempenho e rentabilidade, a ambiência e o bem-estar animal, instalações e equipamentos são pontos de atenção da produção aviária, mas não são foco deste artigo (VASCONCELOS MC, et al., 2021).

Esse breve exposto denota o maior grau de modernização na atividade produtiva, com grande automação e implementação tecnológica das etapas dessa indústria, que se desenvolve, nas últimas décadas, incorporando melhoramentos genéticos e nutricionais, novos processos, novas tecnologias voltadas à produção, instalações e equipamentos, sanidade animal, novos métodos organizacionais e divulgação, para atender a demanda (mercado interno ou externo) (VASCONCELOS MC, et al., 2021).

\section{Relação entre Nutrição e Sistema Imunológico das Aves}

A excelência da genética das aves e o manejo sanitário associados à alta capacidade do sistema imune resultam na evolução da avicultura, superando os principais desafios dos sistemas de produção (BASSINSS e SILVA CL, 2017).

Silva SRG, et al. (2013) destacam a nutrição animal para melhoria da produtividade, em consonância ao bem-estar e à capacidade imunitária das aves. Nesse sentido, tem-se a imunonutrição como a solução ideal para aumentar a resistência do organismo a doenças, empregando-se nutrientes imunomoduladores.

Conceitua-se 'imunonutrição' como a ciência que regula as atividades do sistema imune dos animais por meio de nutrientes ou de alimentos específicos (em devidas quantidades), no intuito de estimular a resistência a doenças e eliminar outros problemas, o que constitui alternativa ef etiva que auxilia a manutenção do estado saudável das aves (SILVA SRG, et al., 2013).

Já o termo 'imunomodulação' é mais recomendado para se referir a um produto ou microrganismo que permita a melhoria da resposta imune das aves, sobretudo das mucosas, para a mobilização de células imunes. Destacam-se como imunomodularores os prebióticos, probióticos, extratos de plantas, vitaminas e minerais (CARDOSO ALS e CASTIGLIONI TESSARI EN, 2015). Silva SRG, et al. (2013) consideram os minerais e as vitaminas os principais imunomoduladores.

Nessa perspectiva, o efeito positivo de uma suplementação alimentar, a partir de nutrientes e de características dietéticas, que podem modular a susceptibilidade das aves com relação a doenças infecciosas, conforme ingredientes utilizados, pode apresentar importância crítica na nutrição de aves (SILVA SRG, et al., 2013).

Do mesmo modo, Cardoso ALS e Castiglioni Tessari EN (2015) asseguram que a nutrição constitui ferramenta para modular o sistema imunológico das aves, com a finalidade de produzir um estado ideal de imunidade, visto que as alterações do sistema imunológico demandam energia e diversos nutrientes para a formação de células e outras substâncias envolvidas no sistema de defesa das aves.

Conforme mencionado, para determinar a imunocompetência e a maior resistência a desafios sanitários nos animais, baseia-se na imunomodulação, através da nutrição, a fim de demonstrar os efeitos de quantidades de vitaminas, minerais e aminoácidos sobre a imunidade humoral e celular (CARDOSO ALS e CASTIGLIONI TESSARI EN, 2015).

Silva SRG et al. (2013) explicam um premix de nutrientes essenciais composto por minerais e vitaminas desenvolvido para imunomodulação. Sua ação já começa no trato gastrointestinal, entrada natural de 
patógenos externos oriundos da ingestão de micróbios, e cuja mucosa e microbiota apresentam defesas imunológicas importantes. Assim, a imunomodulação propicia aumento da resistência das aves, refletindo no aumento de produtividade e, consequentemente, no lucro (SILVA SRG, et al., 2013).

Os principais imunomoduladores são os minerais e as vitaminas, cuja suplementação pode fornecer todos os nutrientes requeridos para que as aves cresçam mais rápido e produzam o máximo de carne e ovos (SILVA SRG, et al., 2013).

De acordo com Norup LR, et al. (2008), há nutrientes que participam mais expressivamente para deposição de carne (tais como metionina, treonina, triptofano e arginina, vitaminas e minerais), e de forma menos significativa para funções de def esa (como, por exemplo, a lisina). Contudo, Cardoso ALS e Castiglioni Tessari EN (2015) ressalvam que, em certos casos, as dietas e suplementações auxiliam no desempenho produtivo, mas não favorecem a máxima proteção imunológica. Assim, a deficiência crônica severa de micronutrientes (vitaminas e minerais) torna-se mais debilitante ao sistema imunológico em comparação aos níveis de energia e de proteína.

A literatura mais recente, disponibilizada em bancos de dados eletrônicos, mostra que diversos experimentos foram realizados em condições nacionais, com o objetivo de analisar ef eitos de diferentes planos nutricionais sobre o desempenho produtivo e econômico de aves de corte. Quanto a esse desempenho, os programas de alimentação incluem os nutrientes essenciais descritos a seguir.

\section{Nutrientes Essenciais}

Segundo Pessoa GBS, et al. (2012), o programa de alimentação deve ser desenvolvido conforme exigências nutricionais de cada fase de crescimento, consoante protocolo alimentar para o planejamento econômico e redução de custos. No Brasil, esse programa se divide em até cinco fases, fornecendo os nutrientes necessários em cada etapa, considerando-se as funções produtivas economicamente fundamentais: ganho de peso, consumo de ração, conversão alimentar e rendimento de cortes.

Os autores ainda advertem que a má nutrição prejudica o sistema imunológico das aves, tornando-as frágeis e suscetíveis a infecções gastrointestinais, atrasando o ganho de peso e gerando maiores custos de produção. Já a dieta alimentar de boa qualidade modula a propensão a infecções e inflamações nas aves, promovendo a absorção adequada dos nutrientes pelo trato gastrointestinal, além de assegurar a conversão alimentar e o ganho de peso (PESSOA GBS, et al., 2012).

Os nutrientes essenciais incluem aminoácidos (proteínas), vitaminas, minerais, ácidos graxos e água. Mesmo não sendo nutrientes, as gorduras e os carboidratos são responsáveis por proporcionar a energia da dieta, configurando componentes essenciais para o desenvolvimento metabólico animal (CARDOSO ALS e CASTIGLIONI TESSARI EN, 2015).

As vitaminas $A, D, E$ e C são os principais imunomoduladores, para os autores supracitados. Igualmente, citam-se os minerais: cromo, selênio e zinco que também podem estimular o sistema imunológico, além de melhorar o desempenho e bem-estar das aves (STRATEN C, 2011).

Trata-se de micronutrientes atuantes em inúmeros processos metabólicos do organismo e essenciais para ótima saúde e desempenho do animal (FÉLIX AP, et al., 2009). Quanto à classificação, as vitaminas podem ser solúveis em gordura ( $A, D, E$ e $K$ ) e as solúveis em água (do grupo $B$ e C) (EMPRESA BRASILEIRA DE PESQUISA AGROPECUÁRIA (EMBRAPA), 2021).

Como o organismo das aves é incapaz de sintetizar todas as vitaminas exigidas, destaca-se a necessidade de inclusão de microquantidades de nutrientes nas dietas. Caso contrário, haverá deficiência de uma ou mais vitaminas, causando distúrbios metabólicos e queda de produtividade, crescimento e desenvolvimento de doenças (BASTOS MCA, 2008).

Quanto aos minerais, estes podem ser fornecidos complexados (forma orgânica), sendo mais eficientemente absorvidos e prontamente armazenados por tempo mais longo que os inorgânicos. Nesse âmbito, reforça-se a importância do zinco, cromo, selênio, e do ferro (CARDOSO ALS e CASTIGLIONI 
TESSARI EN, 2015). Zinco orgânico, cobre e selênio (associados à vitamina E), e as vitaminas A, D e E são exemplos de suplementação, no complemento de rações, para auxiliar a defesa do sistema imunológico (STRATEN C, 2011).

Podem ser divididos em macrominerais (presentes em maiores quantidades na dieta - $\mathrm{Ca}, \mathrm{P}, \mathrm{K}, \mathrm{Na}, \mathrm{Cl}$ e $\mathrm{S})$ e microminerais ( $\mathrm{Cu}, \mathrm{Zn}, \mathrm{Fe}, \mathrm{Mn}, \mathrm{I}, \mathrm{Mo}, \mathrm{Se}, \mathrm{Cr}$ ). Sua função é manter o metabolismo fisiológico e suas formas mais empregadas são os quelatos, compostos pela reação do mineral com um hidrolisado de aminoácidos e/ou peptídios, e os minerais orgânicos constituídos pela incorporação biossintética de um mineral em um aminoácido (EMBRAPA, 2021).

Fosou A, et al. (2016) explicam que o zinco integra a estrutura de enzimas ampliando as respostas imunológicas; o cobre age com poder antioxidante, permitindo a produção adequada de anticorpos, e reduzindo a susceptibilidade a infecções. Por sua vez, o selênio e a vitamina $E$ participam em enzimas da cadeia de ações do sistema imunológico. A vitamina A maximiza a conversão alimentar, propicia o crescimento da ave e permite a expressão do sistema imunológico. A vitamina $D$ atua junto a receptores de inúmeros tipos de células, incluindo os macrófagos, incrementando a resposta imunológica das aves. Isoladamente, a vitamina $\mathrm{E}$ interfere na multiplicação celular do sistema imunológico, ao produzir anticorpos e agir como antioxidante para integridade da membrana celular (FOSOU A, et al., 2016).

lgualmente, aminoácidos precisam ser fornecidos em quantidades adequadas. Isso significa formular uma dieta baseada na proteína ideal, a fim de suprir o mínimo, para se obter melhor crescimento. Deve-se, pois, considerar a proteína do ingrediente, o tipo da fibra, os fatores antinutricionais, o processamento (temperatura, pressão), a metodologia de pesquisa e a fase de vida da ave (EMBRAPA, 2021).

Pessoa GBS, et al. (2012) descrevem os principais aminoácidos limitantes (lisina, metionina, treonina, triptofano e valina) como comercialmente disponíveis e mais competitivos quanto a custos. Já a ingestão do sódio (na quantidade correta) auxilia absorção de monossacarídeos e aminoácidos pelo trato gastrointestinal, pois a bomba de sódio permite o transporte desses elementos, possibilitando a conversão dos alimentos e o ganho de peso (CARDOSO ALS e CASTIGLIONI TESSARI EN, 2015).

A energia é um componente restritivo, apresentando alto custo na formulação da dieta. Em geral, reduzse a energia da dieta para diminuir o seu custo, sem causar prejúzo no desempenho das aves. A quantidade varia conforme a fase produtiva, o sexo, a condição ambiental, a capacidade digestiva e o consumo diário (EMBRAPA, 2021).

Cardoso ALS e Castiglioni Tessari EN (2015) defendem que o conhecimento das exigências energéticas auxilia no ajuste dos demais nutrientes, resultando numa formulação adequada. As gorduras das dietas são compostas pelos ácidos graxos (linoleico e alfa-linolênico), fundamentais para as aves, por atuarem no metabolismo (membrana celular e hormônios) e fornecerem energia. Origina-se de ingredientes ou de gorduras adicionadas à dieta (LEDUR MC, et al., 2011).

Vale ressaltar que a falta de ácidos graxos pode induzir o comprometimento da integridade das membranas, além de causar descamação da pele, redução à resistência patológica, e gerar distúrbios reprodutivos nas aves (EMBRAPA, 2021).

\section{Melhoramento Genético}

Importante reforçar que o setor aviário de corte é importante fornecedor mundial de proteína animal, está em constante evolução; e seu avanço diretamente associado à melhoria genética das aves, através de uma seleção baseada em características de produtividade (SELL-KUBIAK E, et al., 2017).

Martins JMS, et al. (2012) confirmam que a cadeia produtiva de frangos de corte no Brasil sofre alterações e passa por modernizações, promovendo redução de custos e de aumento produtivo, mantendo a competitividade e o desempenho econômico em nível mundial, contribuindo significativamente para a economia brasileira.

Como estratégia de melhoramento, destaca-se a seleção de linhagens de aves com características superiores (maior conversão alimentar, tamanho de peito, resistência a infecções etc.). Assim, objetiva-se o 
incremento genético sobre as características desejadas, com a finalidade de aprimorar a relação "Custo $X$ Benefício" (MARTINS JMS, et al., 2012).

Visando à melhoria do material genético das aves de corte, busca-se identificar linhagens com características superiores, selecionando-se animais de melhor desempenho. Segundo Costa LS (2015), a avicultura brasileira ainda depende da tecnologia genética estrangeira, mesmo existindo alguns programas nacionais de produção de material genético.

Contudo, sabe-se que a constante utilização de tecnologias genéticas avançadas possibilitou a redução de custos e o aumento da produtividade, além de propiciar a diversificação de linhagens para atender a uma maior fatia de mercado. Nesse contexto, são necessárias a variabilidade genética dos animais e a hibridação (pois a melhoria genética depende da divergência genética dos progenitores, devendo ser introduzidos novos genótipos no plantel) (LEDUR MC, et al., 2011).

Quanto à alteração na composição genotípica da população, foca-se no ganho genético sobre determinada característica ou sobre um conjunto de características de interesse econômico. As principais qualidades almejadas advêm de diversos pares de genes que, combinados, permitem os resultados esperados (MARTINS JMS, et al., 2012).

Características como peso corporal, conversão alimentar, viabilidade geral e específica, rendimento e gordura na/da carcaça, empenamento e ausência de defeitos são enfatizadas como viabilidade geral e específica. Da mesma forma, selecionam-se melhor fertilidade, eclodibilidade e produção de ovos incubáveis (SELL-KUBIAK E, et al., 2017).

Boaretto TN (2009) confirma que pesquisas em biologia molecular permitem o sequenciamento dos genomas e o entendimento sobre as funções específicas de genes distintos, proporcionando avanços nos programas de melhoramento genético.

Para alcançar as melhores aves para reprodução, é preciso selecionar indivíduos com desempenho máximo de suas carcaças, características de baixa herdabilidade, tais como resistência a inf ecções/doenças, ausência de problemas metabólicos, maior produção de ovos e alto índice de reprodução, a partir da implementação de programas de melhoramento genético, devidamente guiados pelo sequenciamento genético (GROSSO JL, 2010).

Devido ao grande desenvolvimento genético que ocorre nas linhagens avícolas, a suplementação de nutrientes aumenta o estado nutricional do embrião, interferindo positivamente no desempenho e no desenvolvimento do sistema imune de pintos de corte. O ef eito da suplementação de um complexo enzimático mostrou resultados satisfatórios no ganho de peso e na conversão alimentar de aves no período de 1 a 42 dias (PESSOA GBS, et al., 2012).

Mudanças tecnológicas na área de nutrição/produção de aves de corte requer atualização profissional, adaptação de novas tecnologias às condições brasileiras, somando-se estratégias nutricionais para melhorar aproveitamento dos nutrientes, aumentar a produtividade dos animais e diminuir excreção de nutrientes. Algumas técnicas ainda reduzem custos de produção (PESSOA GBS, et al., 2012).

Pesquisas constantes em nutrição das aves aperf eiçoa um sistema imunocompetente, por meio da seleção de aves melhoradas geneticamente e da manutenção sanitária, contribuindo para que as granjas alcancem os resultados esperados (LEDUR MC, et al., 2011).

\section{CONSIDERAÇÕES FINAIS}

A nutrição é uma ferramenta capaz de possibilitar respostas mais eficientes na busca por melhores produtos genéticos, tornando de suma relevância a análise constante dos índices de desempenho zootécnico de aves de corte, para nortear programas de seleção e melhoramento genético de linhagens. O processo imunológico das aves de corte afeta diretamente a produção, pois quanto melhor o sistema imunológico melhor o desenvolvimento dessas aves. Portanto, a imunomodulação auxilia na manutenção do estado 
saudável das aves, porfacilitar o conhecimento das necessidades nutricionais que maximizam o desempenho produtivo e reprodutivo das aves.

\section{REFERÊNCIAS}

1. BASSI NSS, SILVA CL. Oportunidades e desafios em pd\&i na cadeia produtiva de frangos de corte. Agropensa, Embrapa Suínos e Aves. Universidade Tecnológica Federal do Paraná (UTFPR), 2017.

2. BASTOS MCA. Bioquímica básica: introdução à bioquímica dos hormônios, sangue, sistema urinário, processos digestivos, absortivos e micronutrientes. Rio de Janeiro: Interciência, 2008;213 p.

3. BOARETTO TN. Melhoramento Genético em Frangos de Corte. Revista Formação Informação Zootecnia, 2009; 1(1).

4. CARDOSO ALS, CASTIGLIONI TESSARI EN. Interação entre imunidade e nutrição das aves: revisão de literatura. Revista Científica de Medicina Veterinária. 2015;24.

5. COSTA LS. A indústria de frango de corte no mundo e no Brasil e a participação da indústria avícola paranaense neste complexo. Ciências Sociais em Perspectiva, 2015;14(27):319-341.

6. DEMATTÉ FILHO LC, MARQUES PEM. Dinâmica Tecnológica da Cadeia Industrial da Avicultura Alternativa: Multifuncionalidade, Desenvolvimento Territorial e Sustentabilidade. Segurança Alimentar e Nutricional, 2011; 18(2): 1-11.

7. EMPRESA BRASILEIRA DE PESQUISA AGROPECUÁRIA (EMBRAPA). Produção frangos de corte. Disponível em: https://www.embrapa.br/suinos-e-aves/publicacoes. Acesso em: 10 jul. 2021.

8. FÉLIX AP, et al. Níveis vitamínicos para frangos de corte. Ciência Rural. Santa Maria, 2009;39(2): 619-626.

9. FOSOU A, et al. Desempenho, imunidade e respostas fisiológicas de frangos de corte às variações sequenciais de energia e proteína da dieta. Ciência Avícola, 2016.

10. GROSSO JL. Estimação dos Valores Genéticos e Econômicos para as Características sob Seleção. Produção Animal Avicultura, 2010;4(40):37.

11. JOHANN M, et al. Avitaminoses em aves de criação: revisão de literatura. XVII Seminário Interinstitucional de Ensino Pesquisa e Extensão, 2012.

12. LEDUR MC, et al. Novos Rumos da Genética no Desenvolvimento Avícola. Avicultura Industrial, 2011;102(1): $12-16$.

13. MARTINS JMS, et al. Melhoramento genético de frangos de corte. Pubvet, 2012: 6(18).

14. NORUP LR, et al. Effect of mild heat stress and mild infection pressure on immune responses to an E. coli infection in chickens. Journal Animal Science, 2008;2: 265-274.

15. PESSOA GBS, et al. Novos conceitos em nutrição de aves. Rev. Bras. Saúde Prod. Anim., 2012; 13(3):755-774.

16. SELL-KUBIAK E, et al. Aspectos Genéticos da eficiência alimentar e redução da pegada ambiental em frangos de corte. Journal of Applied Genetics, 2017;36 (2).

17. SILVA ICM, RIBEIRO AML. Interação entre a nutrição e a imunologia em aves. Avisite Produção Animal - Avicultura, 2009;22: 18-25.

18. SILVA SRG, et al. Fundamentos da imunonutrição em aves. Revista Eletrônica Nutritime. Fundamentos da imunonutrição em aves, 2013;10(1):2154-2172.

19. STRATEN C. Uso de vitaminas para melhorar a produção avícola. 2011. Disponível em: https://www.wattagnet.com/articles/10526-using-vitamins-to-improve-poultry-production. Acesso em:15 nov. 2020.

20. VASCONCELOS MC, et al. Caracterização das tecnologias e inovação na cadeia produtiva do frango de corte no Brasil. VII Seminário de Pesquisa Interdisciplinar. Unisul, Universidade Tecnológica Federal do Paraná(UTFPR), 2021. 